\title{
Irrelevant Conjunction and the Ratio Measure or Historical Skepticism
}

\author{
J. Brian Pitts \\ Department of Philosophy, Department of Physics, and \\ Reilly Center for Science, Technology and Values \\ University of Notre Dame \\ Notre Dame, Indiana 46556 USA \\ jpitts@nd.edu, 574 904-1177
}

May 23, 2011

\begin{abstract}
It is widely believed that one should not become more confident that all swans are white and all lions are brave simply by observing white swans. Irrelevant conjunction or "tacking" of a theory onto another is often thought problematic for Bayesianism, especially given the ratio measure of confirmation considered here. It is recalled that the irrelevant conjunct is not confirmed at all. Using the ratio measure, the irrelevant conjunction is confirmed to the same degree as the relevant conjunct, which, it is argued, is ideal: the irrelevant conjunct is irrelevant. Because the past's really having been as it now appears to have been is an irrelevant conjunct, present evidence confirms theories about past events only insofar as irrelevant conjunctions are confirmed. Hence the ideal of not confirming irrelevant conjunctions would imply that historical claims are not confirmed. Confirmation measures partially realizing that ideal make the confirmation of historical claims by present evidence depend strongly on the (presumably subjective) degree of belief in the irrelevant conjunct. The unusually good behavior of the ratio measure has a bearing on the problem of measure sensitivity. For non-statistical hypotheses, Bayes' theorem yields a fractional linear transformation in the prior probability, not a linear rescaling, so even the ratio measure arguably does not aptly measure confirmation in such cases.
\end{abstract}




\section{Introduction}

The problem of irrelevant conjunction or "tacking" is often presented as a difficulty for Bayesian confirmation theory in general or for the ratio measure of confirmation in particular. A number of authors find it evident that a theory $T_{1}$ ought not to be evidence for the conjunction $T_{1} \& T_{2}$ for an irrelevant conjunct $T_{2}$. If that desideratum is impossible, at least they want to reduce the damage by having $T_{1} \& T_{2}$ confirmed less than $T_{1}$. Suppose that, if a definite example is required, $T_{1}$ is the claim that all swans are white, while $T_{2}$ is the theory that all lions are brave. ${ }^{1}$ The irrelevance is obvious: one cannot learn about the courage of lions simply by studying the colors of swans. I take this sort of example to be illuminating regarding what one means by irrelevant conjunction. Yet Bayesian confirmation theory, with various measures of confirmation, including the ratio measure, implies that observing white swans confirms $T_{1} \& T_{2}$ : one becomes more confident that all swans are white and all lions are brave, simply by observing white swans. Can one thereby become more confident that all lions are brave by observing white swans? Obviously the right answer is "no." Such a result also follows, fortunately, from Bayesian confirmation theory. But is that enough to resolve the worry, or is there some further problem about the conjunction's being confirmed?

Whether there is any problem of irrelevant conjunction depends largely on one's intuitions about measures of confirmation and about irrelevant conjunctions. Some authors are attracted to the ratio measure, according to which there is no problem of irrelevant conjunction. Irrelevant conjunctions are confirmed, indeed confirmed just as much as the relevant conjunct is, and that is accepted as a result, not an absurdity. (Of course the posterior probabilities, like the priors, are not equal in general, so absurdity isn't obvious.) Other authors are attracted to other measures of confirmation and hold that it is problematic that irrelevant conjunctions are confirmed. The ideal for many such authors, apparently, is that irrelevant conjunctions not be confirmed at all. That ideal is incompatible with Bayesianism. Others wish for irrelevant conjunctions to be confirmed, but less than is the relevant conjunct [Rosenkrantz, 1994]. Many settle for this view who would have preferred the stronger ideal of no confirmation.

The dialectical situation is a standoff. It is not the case that non-ratio Bayesians have generated some result that pro-ratio Bayesians need to parry, as is often thought (e.g., [Fitelson, 2002]). The ratio proponent is therefore rationally free to present the ratio view as an attractive option. But merely expressing one's intuitions and inviting others to join in does usually not make much progress in a longstanding debate. This paper invites the reader to reconsider intuitions indicating that there is any such problem, by displaying how well the ratio measure fits the mathematics of Bayesianism mathematically, whereas other measures are often motivated by an intuition (that irrelevant conjunctions should not be confirmed) that is incompatible with Bayesianism. Furthermore,

\footnotetext{
${ }^{1}$ Of course one now knows that some swans are black, and it doesn't matter how many lions really are brave.
} 
confirmation measures that aren't attuned to the mathematics of Bayes' theorem in the way that the ratio measure is, need not stay closely in step with the changes in probabilities. This fact (not an independent intuition in favor of some confirmation measure or of its numerical behavior in specific cases) seems to me to be the proper import of the cases of "unintuitive confirmation" that have been proposed in favor of the ratio measure [Schlesinger, 1995]. While there is parity in the unintuitive confirmation arguments against and for the ratio measure [Fitelson, 1999], the rootedness of the ratio measure in Bayes' theorem breaks the symmetry. Those are already reasons to favor the ratio measure.

This paper also proposes an example where the ratio measure behaves plausibly, whereas the ideal of not confirming irrelevant conjunctions generates the absurd result that present evidence doesn't confirm theories about past events. Bayesianism does not permit that absurd result, however, because it requires irrelevant conjunctions to be confirmed to some degree. The weaker goal of having irrelevant conjunctions confirmed less than the relevant conjunct has the effect of making confirmation depend on the prior probability of the theory in question, thus importing massive subjectivity into the degree of confirmation for subjective Bayesians.

One thus has a number of reasons to favor the ratio measure and the related view that there is no problem with confirming irrelevant conjuncts over (most) alternative measures and the associated view that it is problematic for irrelevant conjunctions to be confirmed:

1. The ratio measure is motivated by the mathematical form of Bayes' theorem, whereas most of the competition is not.

2. Insofar as a measure of confirmation is not motivated by Bayes' theorem, there seems to be nothing to prevent confirmation from getting quite out of line with the trend of the posterior probabilities, making confirmation an idle wheel on the Bayesian machinery.

3. The view that irrelevant conjunctions should not be confirmed leads to a formal profession of historical skepticism, according to which present evidence doesn't confirm claims about past events at all.

4. The view that irrelevant conjunctions should not be confirmed is incompatible with Bayesianism, forcing its proponents to settle for only partial realization of their ideal.

5. That partial realization, that irrelevant conjunctions should be confirmed noticeably less than the relevant conjunct, leads to a formal profession of historical subjectivism, according to which present evidence confirms claims about past events to a degree that might be low and that depends strongly on one's prior probability for the irrelevant conjunct.

Concerning historical skepticism or subjectivism, there might well be other reasons for such views about the past in view of Hume's induction skepticism 
[Howson, 2000] - real skepticism, not a formal profession of skepticism potentially belied by high probability assignments, for example. ${ }^{2}$ But it is surprising and undesirable for a principle of confirmation theory to imply or encourage skepticism or subjectivism regarding confirmation of claims about the past.

Given that there isn't really any problem of irrelevant conjunction, using the ratio measure, it is useful to consider why many people think that there is. It is suggested that one real problem in the neighborhood, which might encourage faulty intuitions, is whether the irrelevant conjunct is confirmed. It isn't, whether using the ratio measure or some other. Another possible source of confusion is the special consequence condition on confirmation, which Bayesians must reject.

But not all is well for the ratio measure as the one true measure of confirmation in all contexts. Attention to which probabilities are epistemologically primitive indicates that, for non-statistical hypotheses, Bayes' theorem involves not multiplying the prior probability by a number to get the posterior, but acting on the prior with a fractional linear transformation with a parameter involving the ratio of the likelihoods $P(E \mid T)$ and $P(E \mid \neg T)$.

\section{Irrelevance}

What, mathematically speaking, is it for $T_{2}$ to be irrelevant to $T_{1}$ and to the evidence $E$ for or against $T_{1}$ ? Given the vagueness of the problem of irrelevant conjunction in many discussions, it might be appropriate simply to read off the irrelevance condition as whatever it takes to avoid confirming $T_{2}$. But one also has some intuitions about probabilistic irrelevance; one hopes that intuitive expectations about probabilistic irrelevance and the condition needed to avoid confirming $T_{2}$ are similar. Concerning the intuitions about probabilistic irrelevance of the irrelevant conjunct, this irrelevance should manifest itself mathematically in probabilistic independence of the two theories:

$$
P\left(T_{2} \mid T_{1}\right)=P\left(T_{2}\right) .
$$

\footnotetext{
${ }^{2}$ On the cultural significance of justifying induction, see Gerhard Schurz [Schurz, 2008]. Schurz's justification of ordinary induction, which is meta-inductive in the tradition of Hans Reichenbach, depends on our knowing that ordinary induction has worked better than any alternative so far. However, it is unclear, in advance of justifying induction, how one knows that ordinary induction has been more predictively successful that any rival method. Where historical facts are absent or controversial (reconstructed from rival inductive methods), every method judges itself successful [Burks, 1953]. If the past is not recalled perfectly and uncontroversially, then there is room for proponents of methods that disagree occasionally with ordinary induction to claim that their methods have been most successful and hence justified instead of ordinary induction, especially if their methods align with allegedly historical reports. Such methods are an example of the "asymptotic rules" that most seriously afflicted Reichenbach's justification of induction [Salmon, 1974]. They are also probably the only kind of rival method that non-philosophers have ever entertained, providing the real competition for ordinary induction in the intellectual history of the Enlightenment, for example.
} 
One would also expect $T_{2}$ to be independent of the evidence for or against $T_{1}$ : for all $E$ such that $P\left(E \mid T_{1}\right) \neq P(E)$, one has

$$
P\left(T_{2} \mid E\right)=P\left(T_{2}\right) .
$$

One would expect $T_{2}$ to be irrelevant not merely to $T_{1}$ and to $E$, but also to their conjunction:

$$
P\left(T_{2} \mid T_{1} \& E\right)=P\left(T_{2}\right)
$$

for all such $E$. Is this condition independent?

One can show that this third condition is mathematically independent of the other two. The following example (momentarily suspending talk of swan color and lion courage) satisfies the first two independence conditions, violates the third independence condition, and has $E$ confirm $T_{1}$. Consider an automotive show with 16 cars: 6 fast blue Fords, two slow red Fords, 6 fast red Chevrolets, and two slow blue Chevrolets. Let a car be chosen at random, and let $T_{1}$ be that the car is a Ford, $E$ that it is blue, and $T_{2}$ that it is fast. One then has

$$
\begin{array}{r}
P\left(T_{2} \mid T_{1}\right)=P(\text { fast } \mid \text { Ford })=6 / 8=P\left(T_{2}\right)=P(\text { fast })=12 / 16, \\
P\left(T_{2} \mid E\right)=P(\text { fast } \mid \text { blue })=6 / 8=P(\text { fast })=12 / 16, \\
P\left(T_{2} \mid T_{1} \& E\right)=P(\text { fast } \mid \text { Ford } \& \text { blue })=6 / 6 \neq P\left(T_{2}\right)=P(\text { fast })=12 / 16, \\
P\left(T_{1} \mid E\right)=P(\text { Ford } \mid \text { blue })=6 / 8>P\left(T_{1}\right)=P(\text { Ford })=1 / 2 .
\end{array}
$$

Thus the irrelevance of the irrelevant conjunct needs to involve $T_{2}$ 's being independent of $T_{1}$, of $E$, and of $T_{1} \& E$. It is easy to show that $P\left(T_{2} \mid T_{1} \& \neg E\right)=P\left(T_{2}\right)$ is not an independent condition; a precisely analogous calculation shows that $P\left(T_{2} \mid \neg T_{1} \& E\right)=P\left(T_{2}\right)$ is also already implied. Likewise $P\left(T_{2} \mid \neg T_{1} \& \neg E\right)=$ $P\left(T_{2}\right)$ is a consequence of the three independence conditions above.

\section{The Ratio Measure}

The ratio measure of confirmation (or something equivalent) has been praised by some authors [Howson, 1983, Horwich, 1993, Schlesinger, 1995, Milne, 1995, Milne, 1996, Kuipers, 2000] and criticized by others [Rosenkrantz, 1983, Gillies, 1986, Fitelson, 2001, Eells and Fitelson, 2002, Hawthorne and Fitelson, 2004, Crupi and Tentori, 2010]. In the former group, Peter Milne is perhaps the most ambitious in claiming to prove that the (log) ratio measure is the one true measure of Bayesian confirmation [Milne, 1996]. For Milne, the ratio measure (or rather its logarithm - there is no need here to distinguish measures that are obviously equivalent in the sense of quantitative interconvertibility) follows from an axiom about certain kinds of probabilistic independence. The treatment is perhaps a bit quick for those who are antecedently suspicious of the ratio measure and even those not antecedently sympathetic toward it. Seeing that the ratio measure is uniquely appropriate in dealing with the problem of irrelevant conjunction (at least among alternatives 
considered by Branden Fitelson) might help to motivate what Milne takes as axiomatic.

If one defines confirmation using the ratio measure

$$
r(T, E)=_{\text {def }} \frac{P(E \mid T)}{P(E)},
$$

which of course is equal to $\frac{P(T \mid E)}{P(T)}$ (apart from extreme cases - a qualification that I will ignore most of the time, but see [Festa, 1999] and later in this section), it turns out that evidence $E$ for $T_{1}$ is also evidence for the conjunction $T_{1} \& T_{2}$ in cases where $T_{2}$ is irrelevant to $T_{1}$ (and $T_{2}$ is logically consistent with $T_{1}$ ). Using the independence condition $P\left(T_{2} \mid T_{1} \& E\right)=P\left(T_{2}\right)$, one calculates that

$$
P\left(T_{1} \& T_{2} \mid E\right)=P\left(T_{2} \mid T_{1} \& E\right) P\left(T_{1} \mid E\right)=P\left(T_{2}\right) P\left(T_{1} \mid E\right),
$$

which is encouraging. The ratio measure becomes

$$
\begin{array}{r}
r\left(T_{1} \& T_{2}, E\right)=\frac{P\left(E \mid T_{1} \& T_{2}\right)}{P(E)}=\frac{P\left(T_{1} \& T_{2} \mid E\right)}{P\left(T_{1} \& T_{2}\right)}=\frac{P\left(T_{2} \mid T_{1} \& E\right) P\left(T_{1} \mid E\right)}{P\left(T_{2} \mid T_{1}\right) P\left(T_{1}\right)}= \\
\frac{P\left(T_{2}\right) P\left(T_{1} \mid E\right)}{P\left(T_{2}\right) P\left(T_{1}\right)}=\frac{P\left(T_{1} \mid E\right)}{P\left(T_{1}\right)}=\frac{P\left(E \mid T_{1}\right)}{P(E)}=r\left(T_{1}, E\right),
\end{array}
$$

making use of $P\left(T_{2} \mid T_{1} \& E\right)=P\left(T_{2}\right)$ and $P\left(T_{2} \mid T_{1}\right)=P\left(T_{2}\right)$. While evidence $E$ that confirms $T_{1}$ also confirms the conjunction $T_{1} \& T_{2}$, it looks as though $T_{2}$ is merely going along for the ride, as an irrelevancy should.

To be sure that $T_{2}$ is not getting confirmed by $E$, one of course ought to consider the confirmation of $T_{2}$ directly. Using the independence condition $P\left(T_{2} \mid E\right)=P\left(T_{2}\right)$, the ratio measure gives

$$
r\left(T_{2}, E\right)=\frac{P\left(E \mid T_{2}\right)}{P(E)}=\frac{P\left(T_{2} \mid E\right)}{P\left(T_{2}\right)}=1,
$$

the trivial value of no confirmation, so $T_{2}$ is not confirmed by the evidence $E$ for $T_{1}$. That is just what one hoped: one cannot become more confident that all lions are brave by heaping up facts about the coloration of swans. This particular feature does not distinguish the ratio measure from other measures, admittedly. The point is simply that when one is concerned about what clearly is a problem (the confirmation of the irrelevant conjunct), not what might not be a problem at all (the confirmation of the irrelevant conjunction), the ratio measure has no problem. It turns out that the three independence assumptions that followed naturally above from the irrelevance of $T_{2}$ precisely imply that the irrelevant conjunct $T_{2}$ is not confirmed by evidence $E$ for $T_{1}$ and that the confirmation that $E$ provides $T_{1} \& T_{2}$ is wholly due to confirmation of $T_{1}$. All is as one might hope for the ratio measure.

The reader might notice that this little derivation exploits as a virtue a phenomenon that Fitelson has viewed as a vice in his theorem 3 [Fitelson, 2001] and substantially similar theorem 2 [Fitelson, 2002]. A fundamental issue here is what the problem of irrelevant conjunction is, or ought to be. On that question, Fitelson provides, in a transparent notation, 
Theorem 1. If $E$ confirms $H$, and $X$ is confirmationally irrelevant to $H, E$, and $H \& E$ (relative to $K$ ), then $E$ also confirms $H \& B$ (relative to K). [Fitelson, 2002]

Many Bayesians would agree with his next remark:

I claim that this is the "problem of irrelevant conjunction" that Bayesian confirmation theorists should be worrying about. [Fitelson, 2002]

But why think that this fact expressed in his theorem 1 is a problem at all? Some time ago Clark Glymour, who had in mind hypothetico-deductivism but presumably would have made the same point against any Bayesianisms where it applied, judged:

Nor are we greatly impressed by a theory of evidence which claims, for instance, that the Stark effect confirms the conjunction of quantum mechanics and the principle that the president of the Church of Jesus Christ of Latter Day Saints is infallible, all the while reassuring us that the Stark effect does not confirm the latter principle alone. [Glymour, 1980, p. 31]

But why not? I, for one, do not at all mind getting that result from Bayesianism with the ratio measure. Much as Milne proposed in addressing the tacking problem in the context of the (log) ratio measure, it makes sense here to embrace the conclusion that has been proposed otherwise as a reductio [Milne, 1996]. Milne, alas, stops short: having found that the irrelevant conjunction is confirmed to the same degree as the relevant conjunct assuming $P\left(E \mid T_{1}\right)=P\left(E \mid T_{1} \& T_{2}\right)$ (after translation into my notation and suppression of mention of the background information), he notes only that one cannot infer anything about the degree of confirmation of the irrelevant conjunct $T_{2}$ by $E$. He does not explore fully enough what the irrelevance of $T_{2}$ means to show that it, the irrelevant conjunct, is confirmed only to the trivial value ( 1 for the ratio measure, 0 for a log-ratio measure), which is to say, not confirmed.

Confirming the irrelevant conjunction to the same degree as the relevant conjunct and not confirming the irrelevant conjunct at all is a very plausible way for an irrelevant conjunct merely to go along for the ride. For his part, Glymour is unwilling to take a stand against the special consequence condition [Glymour, 1980, pp. 31, 155], a fact that makes clear one reason why he would embrace not the resolution proposed in this paper (besides his not being a Bayesian). But Bayesians generally do reject the special consequence condition, so they will need some other reason to reject this outcome as a flaw rather than a result. Paul Horwich has made this point in a brief verbal form near the end of his book, where he interacts with Glymour's bootstrap account of confirmation, which deliberately has the consequence that "neither evidence for $p$, nor even the discovery of $p$, is sufficient to confirm the conjunction $p \wedge q$." [Horwich, 1982, p. 139] (see also [Horwich, 1983]). But is it desirable to avoid the conclusion that an irrelevant conjunction is confirmed at least to some degree (even if perhaps less than the relevant conjunct)? Horwich comments, pace Glymour, that 
There are indeed times when $T$ is confirmed, even though the evidence is irrelevant to some part $T^{\prime}$ of $T$. But there is nothing in either Bayesianism or the H-D method to suggest that $T^{\prime}$ should be given any credit. Unless we were, mistakenly, to endorse the consequence condition (criticised in Chapter 3), we would not conclude from the fact that $T$ entails $T^{\prime}$, that $T^{\prime}$ is supported by whatever confirms $T$. [Horwich, 1982, pp. 140, 141]

Like Milne, Horwich has made room for the possibility that the irrelevant conjunct is not confirmed, but has not presented probabilistic relations of independence to show that such an attractive possibility is realized. The ongoing reluctance of various other authors to take the ratio measure seriously as giving an adequate, indeed a superior, treatment of the problem of irrelevant conjunction makes it appropriate to be explicit on this point.

Fitelson has made clear that an irrelevant conjunction is confirmed (according to the ratio measure) as strongly as the relevant conjunct, but without taking seriously the idea that a solution of the problem of irrelevant conjunction is thereby obtained. Evidently Horwich's suggestion about what the real problem is, has not made the impact that it deserved. Indeed this sort of view does not even make an appearance in Fitelson's survey of responses to the problem of irrelevant conjunction [Fitelson, 2001]. Moreover, Fitelson has regarded the ratio measure of confirmation as incapable of solving the problem of irrelevant conjunction (see footnotes 28 and 36) [Fitelson, 2001, pp. 17, 21] [Fitelson, 2002]. It is acknowledged as one possible move, though not one appealing to him, in a forthcoming work [Fitelson, 2011]; his calling it bullet-biting suggests that its plausibility has not been recognized. Likewise David Atkinson holds that the problem of irrelevant conjunction, having appeared first for hypothetico-deductivism and having been managed by some Bayesians, is "back in full force" for the ratio measure [Atkinson, 2009]. The difficulty, I suggest, is that what they regard as the problem of irrelevant conjunction (quoted above just under Fitelson's theorem 1), that the conjunction is confirmed, is not a problem at all, but simply a result.

It seems that the two most plausible approaches to dealing with irrelevant conjunctions are the extreme ones: either an irrelevant conjunction is not confirmed at all, as on Glymour's view, because the irrelevant part has no eligible place in the confirmation formalism, or an irrelevant conjunction is confirmed to the same degree as the relevant conjunct, as on the ratio confirmation measure in Bayesianism, because the irrelevant conjunct makes no difference. If the irrelevant conjunct is really irrelevant, then why should the conjunction be confirmed at all, but less well confirmed? Yet this seems to be the typical Bayesian view nowadays [Earman, 1992, Fitelson, 2001, Hawthorne and Fitelson, 2004, Crupi and Tentori, 2010]. Either ineligibility or irrelevance would seem to be the natural options. This view appears to be in line with that of Patrick Maher [Maher, 2004], but not that of Roger Rosenkrantz [Rosenkrantz, 1994]. Regarding some deductive special cases, Rosenkrantz expects no confirmation (which seems to mean that the degree of confirmation is defined and has a value of 
0 ) when $T_{1}$ excludes $T_{2}$, equal confirmation for $T_{1} \& T_{2}$ and $T_{1}$ when $T_{1} \rightarrow T_{2}$ (naturally), and linear interpolation between those endpoints. While those endpoint values are reasonable, they are not the only reasonable possibilities. For the ratio measure, if $T_{1} \rightarrow T_{2}$, then

$$
r\left(T_{1} \& T_{2}, E\right)=\frac{P\left(E \mid T_{1} \& T_{2}\right)}{P(E)}=\frac{P\left(E \mid T_{1}\right)}{P(E)}=\frac{1}{P(E)}=r\left(T_{1}, E\right):
$$

not surprisingly, the degrees of confirmation of $T_{1}$ and of $T_{1} \& T_{2}$ agree. If instead $T_{1} \rightarrow \neg T_{2}$, then

$$
r\left(T_{1} \& T_{2}, E\right) \stackrel{?}{=} \frac{P\left(E \mid T_{1} \& T_{2}\right)}{P(E)},
$$

which is undefined due to conditionalizing on an impossible proposition. Recalling that

$$
\frac{P(E \mid T)}{P(E)}=\frac{P(T \mid E)}{P(T)}
$$

except in extreme cases, and finding difficulty in an extreme case here, one can try again with

$$
r\left(T_{1} \& T_{2}, E\right) \stackrel{?}{=} \frac{P\left(T_{1} \& T_{2} \mid E\right)}{P\left(T_{1} \& T_{2}\right)}=\frac{0}{0},
$$

which is also undefined. Hence "no confirmation" can reasonably mean that the degree of confirmation is undefined, rather than 0. Thus Rosenkrantz's linear interpolation between two finite endpoints, though displaying a sort of mathematical simplicity, is somewhat arbitrary and not clearly better than the ratio measure's behavior, which does not invite simple interpolation.

As Maher notes, making the irrelevant conjunction be confirmed at all, but less than the relevant conjunct, merely reduces the (supposed) problem without eliminating it; he quotes Fitelson [Fitelson, 2002] about merely "soften[ing] the impact" of the problem. But as Maher notes, "a solution that removed the impact altogether would be better." (He goes on to provide a different resolution from the one proposed here.) Of the two 'extreme' views, the proposal that the conjunction is not confirmed at all takes one out of the Bayesian realm, a drastic move. Furthermore, what does one say about almost irrelevant conjunctions? The obvious remaining candidate is that the conjunction is confirmed precisely as much as the relevant conjunct is confirmed. Not only is Fitelson's problem (that the conjunction is confirmed) plausibly not a real problem, but a stronger and ratio-motivated claim is also plausible: the conjunction is confirmed to the same degree as the relevant conjunct. One would expect something irrelevant to make no difference. What else should irrelevance mean? Regarding the confirmation of an irrelevant conjunction, Theo Kuipers finds the behavior of the ratio measure of confirmation "very plausible" [Kuipers, 2000, p. 55].

One should let the mathematics play a guiding role in framing the desiderata. As Henry Finch put the matter quite a while ago [Finch, 1960]: 
Intuitions of adequacy have little force against the imperatives of systems whose usefulness and consequences extend beyond momentary or locally plausible intuition... Better by far than any "feeling" of adequacy concerning a metrical concept is the finding that the concept functions precisely and advantageously in a confirmed metrical theory.

One can find mathematical examples such that, according to intuitions that some people have, some measure or other besides the ratio measure seems more satisfactory. But given the multiplicity of the competing intuitions and examples [Fitelson, 1999], it is difficult to take such examples very seriously. One could perhaps take that cacophony as a reason not to define a quantitative degree of confirmation at all; one could simply use the Bayesian machinery without calling anything the "degree of confirmation," an indifferentist position [Howson, 2000] (c.f. [Steel, 2007]). The serious possibility of not defining any degree of confirmation at all, and instead merely discussing the dynamics of posterior probabilities in light of evidence, shows that any degree of confirmation that isn't rooted in Bayes' theorem has little work to do.

If one of the many competing definitions of degree of confirmation is worthy of the name, it should not only perform in accord with one's intuitions in some favorite cases, but also bear a close connection to Bayes' theorem and the typical conditionalization dynamics of degrees of belief (leaving aside more sophisticated cases requiring Jeffrey conditionalization or perhaps an alternative objective updating scheme). It is not easy to accept the idea that in a quantitative Bayesian confirmation theory involving some mathematical dynamics of degrees of belief, the "degree of confirmation" is well defined but has little or nothing to do with the dynamics of degrees of belief. Even a merely qualitative agreement (which might suffice to blunt absurd results such as the historical skepticism explained below) is a setback for a quantitative confirmation theory. Surely confirmation is not merely something that happens simultaneously with encountering new evidence and revising one's degrees of belief accordingly, or even some process that involves more than encountering new evidence and revising one's degrees of belief accordingly. Rather, confirmation is the process that updating by Bayes' theorem systematizes. The degree of confirmation is either an informative gloss on the process of updating or an idle wheel. Bayes' theorem strongly suggests that the most fruitful concept of confirmation around which one might build a theory is incremental confirmation in a multiplicative sense, or perhaps some more subtle transformation that can be read off the theorem. By contrast the difference measure, for example, ignores the multiplicative character of confirmation that Bayes' theorem suggests.

The fact that $T_{1} \& T_{2}$ is logically stronger than $T_{1}$ should make no difference in the degree of confirmation just because of the multiplicative character suggested by Bayes' theorem. Intuitions that the logically stronger $T_{1} \& T_{2}$ (with $T_{2}$ irrelevant) should be less confirmed by $E$ than is $T_{1}$, like the special consequence condition, are far more plausible for absolute confirmation than for the incremental confirmation at the heart of Bayesianism. Of course $T_{1} \& T_{2}$ typically has 
lower prior and posterior probabilities than $T_{1}$. Why is that not enough? The ratio measure also has the feature [Steel, 2007], which seems advantageous, of tying Bayesianism more closely to the likelihood principle.

It is useful to recall how the ratio measure behaves in special cases where the relevant theory $T_{1}$ entails the evidence $E$, or where $T_{1}$ is inconsistent with $E$. In the first case, $T_{1} \rightarrow E$ gives $P\left(E \mid T_{1}\right)=1$, so

$$
r\left(T_{1}, E\right)=\frac{P\left(E \mid T_{1}\right)}{P(E)}=\frac{1}{P(E)}
$$

and (if also $T_{2}$ is consistent with $T_{1}$ )

$$
r\left(T_{1} \& T_{2}, E\right)=\frac{P\left(E \mid T_{1} \& T_{2}\right)}{P(E)}=\frac{1}{P(E)} ;
$$

whether the equality of these degrees of confirmation is appropriate is difficult to say in isolation from a larger consideration of the issues. If instead $T_{1} \rightarrow \neg E$, falsification or something like it, then

$$
r\left(T_{1}, E\right)=\frac{P\left(E \mid T_{1}\right)}{P(E)}=\frac{0}{P(E)}=0
$$

and, if $T_{2}$ is consistent with $T_{1}$,

$$
r\left(T_{1} \& T_{2}, E\right)=\frac{P\left(E \mid T_{1} \& T_{2}\right)}{P(E)}=\frac{0}{P(E)}=0 ;
$$

hence the 0 degree of ratio confirmation is a sign of falsification or something close.

One might also comment on the symmetry between $T$ and $E$ in the ratio measure. ${ }^{3}$ Is it peculiar that, one might say, $E$ is confirmed by $T$ just as much as $T$ is confirmed by $E$ ? Strictly speaking, that isn't true: generally $T$ and $E$ play different roles, so contexts where one can speak of (observed) $E$ as confirming $T$ are generally not contexts where one can speak of (observed? known?) $T$ as confirming $E$. But it seems that, qualitatively speaking, it is appropriate that if $T$ makes (potential) evidence $E$ look good by predicting it, then (observed) $E$ makes $T$ look good by confirming it. What of the quantitative equality, however? There seems to be nothing unreasonable about having the two amounts be equal. The best reason for accepting equality is that it seems to be the lesson that the Bayesian mathematics wishes to teach in the dynamics of the probabilities. But perhaps one can say a bit more. If the two quantities should grow or shrink together, presumably there should be some strictly increasing function relating them. But it is not easy to think of a non-arbitrary reason for preferring one strictly increasing function over another, apart from the identity $f(x)=x$. If one were to think of $T$ and $E$ as both being theories, neither would confirm the other, because neither would be evidence. But the symmetry between the two theories would be welcome from the standpoint of at least some measures of coherence [Shogenji, 1999, Schupbach, 2011]. Hence what might have seemed peculiar is in fact beneficial.

\footnotetext{
${ }^{3}$ I thank an anonymous referee for posing an issue in this neighborhood.
} 


\section{Historical Skepticism, Subjectivism, or Con- firmation of Irrelevant Conjunctions}

The fact that the ratio measure of confirmation is suggested by Bayes' theorem, whereas most of the competition (such as taking a difference of two probabilities) is not, is evident, but not really news. Likewise the fact that the ideal that irrelevant conjunctions not be confirmed is incompatible with Bayesianism, is not really news either. Such facts are underappreciated and hence worth reiterating, but it would be ideal to show some plainly absurd consequence as a novel reductio ad absurdum of the cluster of views opposing the ratio measure.

One can imagine learning about the past (beyond living memory) by inferring from present physical traces, or from testimonial evidence in old texts presumably rooted in eyewitness testimony, or both. As long as there is no conflict, there is no pressing worry, but what if they do conflict? In the 18th century it was much disputed how the two sorts of evidence ought to be used in reconstructing the geological past [Rappaport, 1997]. The modern view (as in Buffon), displaying a non-Humean confidence in induction [Sloan, 1985] and a rationalistic increased willingness to reject testimonial evidence for preternatural events, tipped the balance towards physical traces over testimonial evidence, in general, in cases of conflict.

However that may be, the ideal that irrelevant conjunctions should not be confirmed would, if taken seriously, lead to a swift overthrow of all confirmation of claims about the past, based on current physical traces. Let theory $T_{1}$ be some claim about the how the past appears to have been, judging by present physical traces. For example, one can take $T_{1}$ to be the claim that the Earth appears to be 4.5 billion years old. Let $E$ be appropriate facts about rock strata, radiometric dating, etc. Let $T_{2}$ be the claim that the Earth really is whatever age it appears to be, an hypothesis of inductive historical realism. Such a principle, despite being widely believed with considerable firmness, seems untestable and, a positivist might say, metaphysical. What matters for present purposes is that $T_{2}$ is an irrelevant conjunct, or very nearly so. Do we have

$$
\begin{array}{r}
P\left(T_{2} \mid T_{1}\right)=P\left(T_{2}\right), \\
P\left(T_{2} \mid E\right)=P\left(T_{2}\right), \\
P\left(T_{2} \mid T_{1} \& E\right)=P\left(T_{2}\right)
\end{array}
$$

for all $E$ bearing on $T_{1}$ ? It seems so... ${ }^{4} T_{2}$ doesn't care if $T_{1}$ involves an apparent age of 4.5 billion years, 6014 years, eternity, 5 minutes, or some other

\footnotetext{
${ }^{4} \ldots$ at least to a very good approximation using most contemporary scholars' priors. In calling $T_{2}$ "metaphysical," one could also deploy a related positivist bugbear, "theological," to $T_{2}$, or at any rate to $\neg T_{2}$. The only reason for doubting the independence conditions that comes to mind, apart from Cartesian evil demons and the like, comes from early modern history of science: if someone were to take seriously Descartes' justification for pretending that the world had formed naturalistically out of chaos as making it easier to do natural philosophy than if one tried to discern how God really created it in mature form [Descartes, 1985, p. 256] (to which the "virtual individuals" gloss on the term representing the influence of preconceived ideas in a Carnapian continuum of inductive methods [Jeffrey, 1980, pp. 2, 3] is
} 
value. Neither does $T_{2}$ care about the specific facts about rock layers, radiometric dating, potsherds from an ancient city, bubble gum stuck to a school desk, or the like. It is often enough recognized that historical antirealism $\neg T_{2}$ is untestable and idle for practical purposes [Gould, 1985, Brush, 1982]. But so is inductive historical realism $T_{2}$, as is rarely noticed except in discussions of the verificationist criterion of meaning [Peirce, 1934, 5.597, p. 417] [Russell, 1948, pp. 445-447] [Ayer, 1952, pp. 101, 102] [Hempel, 1959]. The lack of temporal action at a distance (to borrow a phrase from Elliott Sober) makes the remote past accessible only via the more recent past. Here is one positivist vs. realist dispute that Bayesianism does not resolve (c.f. [Dorling, 1992]).

Now let us deploy the intuition that irrelevant conjunctions should not receive any confirmation. Ideally the rocks would provide no evidence at all for $T_{1} \& T_{2}$, that the Earth looks 4.5 billion years old and is as old as it looks. But that view is logically equivalent to the usual geological view, presumably shared by most non-ratio Bayesians at the moment, that the Earth looks and is 4.5 billion years old. If such non-ratio Bayesians had their way, the rocks, or rather, facts $E$ about them, would provide little or no confirmation for the usual geological view about the age of the Earth, simply as a consequence of confirmation theory. The same would hold, naturally, for any structurally similar argument, not necessarily pertaining to prehistoric events. One could perhaps make a similar move regarding testimonial evidence. This is a novel and implausible path to historical skepticism. It even casts doubt on the value of both sorts of evidence about the past, as opposed to shifting the balance from one side to the other.

This historical skepticism, fortunately, is a paper tiger. That is because confirmation, insofar as its mathematical form is not motivated by Bayes' theorem, is an idle wheel on the Bayesian machinery of the dynamics of numerical degrees of belief in light of evidence. Most non-ratio measures, by virtue of not being motivated by Bayes' theorem, might yield the verdict that the conjunction that the Earth looks 4.5 billion years old and that it is the same age that it looks, is only weakly confirmed by the geological evidence - they will do so insofar as they successfully implement the motivating ideal of avoiding confirming irrelevant conjunctions. But this result is largely verbal, because the Bayesian

analogous), or the anti-rationalist hypothesis of 18th century Catholics like the Jesuit Father Castel [Ellenberger and Carozzi, 1999, pp. 45, 46, 141] (according to whom Noah's Flood was a miracle and ought not to be explained naturally, so that those who try to do so incur the divine chastisement of bad physics), or Leibniz's view that it was for the best that God did not create the world with a longer past [Alexander, 1956, p. 76], or the like. Leibniz's quoting Horace about joining to a human head a horse's neck leaves one to guess the meaning of the analogy, but Leibniz's idea of maximizing variety and simplicity [Blumenfeld, 1995] might be intended. The lower the probability assigned to such ideas, the better the approximation of irrelevance is for $T_{2}$.

Bayesianism, fortunately, does not suffer the burden of dividing hypotheses into those that aren't really theories and those that are genuine theories [Kukla, 1998, Kukla, 2001] (c.f. [Laudan and Leplin, 1991, Hoefer and Rosenberg, 1994]). One can simply punish hypotheses that strike one as deficient or absurd with low priors. The resulting account is more unified than are demarcationist approaches. It also leaves room for disagreement about which theories are worth taking seriously. That bit of flexibility improves the prospects that Bayesianism will be useful in the history of science. 
machinery runs precisely as if one were using the ratio measure, or as if one used no measure of confirmation at all. One might have a high degree of belief in $T_{2}$ and have $T_{1} \& T_{2}$ receive a high posterior probability due to $E$, but still $T_{1} \& T_{2}$ would be described as only slightly confirmed by $E$, while even that modest degree of confirmation would be admitted grudgingly. But whether or not one announces "the hypothesis is highly confirmed!" on receipt of $E$, the main thing that matters is the trend of the posterior probabilities as evidence rolls in. The typical non-ratio proponent is thus faced with the confusing prospect of, perhaps, discerning only weak confirmation while simultaneously watching posterior probabilities rise and become high for certain historical claims. (At least it isn't clear why that cannot happen, and it ought to happen, given the principle of avoiding confirming irrelevant conjunctions.) If the intuitions about confirmation of irrelevant conjunctions of most non-ratio proponents were to have teeth, it would be necessary to have an appropriately matching alternative scheme for updating probabilities.

Does the mediating position, that irrelevant conjunctions ought to be confirmed, but confirmed less than the relevant conjunct, escape trouble? In fact it falls into subjectivism about confirmation. Consider Rosenkrantz's confirmation measure (with some change in notation and suppression of background knowledge), using independence conditions due to the irrelevance of $T_{2}$ as needed:

$$
\begin{array}{r}
d c\left(T_{1} \& T_{2}, E\right)=P\left(T_{1} \& T_{2} \mid E\right)-P\left(T_{1} \& T_{2}\right) \\
=P\left(T_{2} \mid T_{1} \& E\right) P\left(T_{1} \mid E\right)-P\left(T_{2} \mid T_{1}\right) P\left(T_{1}\right)=P\left(T_{2}\right)\left[P\left(T_{1} \mid E\right)-P\left(T_{1}\right)\right] \\
=P\left(T_{2}\right) d c\left(T_{1}, E\right) .
\end{array}
$$

For people who find $T_{2}$ highly plausible, the irrelevant conjunction is confirmed almost as much as the relevant conjunct-no skepticism there. But what if someone, perhaps a taxpaying voting American citizen interested in public education, or perhaps some historical figure who reasoned like Descartes or like Castel, happens to find $T_{2}$ doubtful? At least for subjective Bayesians, intuitive judgments that doubting $T_{2}$ is unreasonable, are inadmissible. People simply have prior probabilities, and one set is as good as another as long as it isn't dogmatic (setting 0 probability on unrefuted contingent claims) and satisfies the probability calculus. According to the subjective Bayesian,

There ain't no good guy,

There ain't no bad guy,

There's only you and me and we just disagree. ${ }^{5}$

One could imagine, for example, someone's doubting $T_{2}$ but accepting its restriction to events after $4004 \mathrm{BC}$, thereby avoiding skepticism about recorded history (or one stream thereof); that seems to have been roughly Descartes' professed view. Induction is not infrequently justified in terms of just getting by in the world. Consider John Kemeny's conclusion:

\footnotetext{
${ }^{5}$ [sic], Billy Dean, "We Just Disagree."
} 
In short, induction cannot be justified. We can only base it on a more or less plausible sounding assumption [involving something like a uniformity of nature claim].... Only in one sense can we justify induction. While our assumption must be taken on faith, we can point out that unless some such assumption were true human life would be impossible. If nature were designed so that plausible inductions invariably turn out to be wrong, the human race would be wiped out soon. [Kemeny, 1959, p. 121]

But absent a time machine, no one needs to get by in the past, still less in the remote pre-human past. Reichenbach's emphasis on successful prediction has a similar practical aspect, as well as a noteworthy past-future asymmetry reflecting the lack of need to get by in the past [Reichenbach, 1938]. No direct practical disadvantage ensues from accepting only the temporally restricted variant of $T_{2}$. Then the degree of confirmation for claims about the past prior to $4004 \mathrm{BC}$, for someone accepting only the temporally restricted relative of $T_{2}$, suffers due to the smallness of $P\left(T_{2}\right)$. One cannot say that claims about the past are objectively well confirmed; one cannot even say that they are intersubjectively well confirmed without an informal survey of people's degrees of belief in $T_{2}$. That is rather embarrassing for a view of confirmation that renounces the systematic elegance of the ratio measure in order to achieve better fit with certain key intuitions.

By contrast, let us deploy the ratio party's view: there is no problem of irrelevant conjunction, because irrelevant conjunctions are confirmed, indeed (incrementally) confirmed to the same degree as the relevant conjunct, and that is a result rather than a problem. Thus $T_{1} \& T_{2}$ benefits from $E$ by having its probability suitably multiplied in accord with Bayesian conditionalization, just as $T_{1}$ does. $T_{1} \& T_{2}$ does not suffer some unspecified and ideally enormous, infinite or subjective penalty for the irrelevance of $T_{2}$. Then the rock layers really do provide the same degree of confirmation that the Earth looks and is 4.5 billion years old as that it looks 4.5 billion years old - in the technical sense of incremental confirmation by having the prior probability suitably multiplied in accord with Bayes' theorem. $T_{2}$ goes along for the ride, so someone who assigns it a high probability can be confident about historical claims based on present traces, and can also say that they are well confirmed. Judgments of confirmation and multiplicative updates in posterior probabilities remain in harmony. The subjectivity of the prior $P\left(T_{2}\right)$ does not disappear - that would require solving Hume's problem of induction [Howson, 2000] and hence is too much to expect of a confirmation theory — but at least it isn't imported into judgments of confirmation. Avoiding a curious sort of historical skepticism or subjectivism is an advantage for the ratio measure and the view that irrelevant conjunctions should be confirmed accordingly. 


\section{Why Has Confirming Irrelevant Conjunctions Seemed Problematic?}

Given that there is no problem of irrelevant conjunction, why have many people thought that there was? It is not always easy, and not necessary, to give a fully satisfactory explanation for why some people have certain faulty intuitions, once the faultiness is shown. But two possible contributing factors might be lingering worries of taint from the irrelevant conjunct as somehow secretly benefiting from evidence for the relevant conjunct, and failure to repudiate clearly the special consequence condition.

It seems plausible that one's view on the confirmation of conjunctions should be derived from two principles: it should be suggested by an adequate confirmation theory, if possible, and it should ensure that the irrelevant conjunct receives no confirmation. I, for one, can hardly imagine having a strong basic intuition about how irrelevant conjunctions ought to fare in confirmation theory. That seems not a lot more appropriate than having a strong basic intuition that in differential calculus, the derivative of the product ought to take some definite form; the most plausible wrong example, perhaps, is that the derivative of the product be the product of the derivatives. There is some initial plausibility (evidently attracting Leibniz himself for a short time [Child, 1920, Cupillari, 2004]), and the proposal is correct for certain examples (though one isn't likely to find them without looking for them), but it is easily seen to be inconsistent with the basic principles of calculus and incorrect for simple examples, such as having one function constant; one could also mention dimensional incorrectness, if the independent variable is not dimensionless. The view that there is a problem of irrelevant conjunction fares only somewhat better: its definition of confirmation is not motivated by Bayes' theorem despite the existence of a measure that is so motivated, judgments of confirmation can get somewhat out of line with the trajectory of posterior probabilities, the intuition that irrelevant conjunctions should not be confirmed is incompatible with Bayesianism and so is only imper-

fectly realized at best, the view yields a curious form of historical skepticism if fully realized, and the view leads to subjectivism about confirmation if partly realized.

The only real problem in the neighborhood of irrelevant conjunction, then, would seem to be whether the irrelevant conjunct is confirmed. It would indeed be disastrous if one could get confirmation for the infallibility for the Mormon president by doing experiments on the Stark effect, to recall Glymour's example, or if one could learn about lion courage by checking swan coloration. It is of the utmost importance not to confuse confirmation of the conjunction with confirmation of the irrelevant conjunct. Rarely is such confusion explicitly made, to be sure. But one can find evidence of occasional wavering. Consider John Norton on an irrelevant conjunction example related to the one deployed above:

Consider a revisionist geology in which the world is supposed created in exactly $4004 \mathrm{BC}$ complete with its fossil record. Standard and revisionist geologies have the same observational consequences, but 
surely we do not think that the fossil record confirms a creation in $4004 \mathrm{BC}$ just as strongly as the ancient earth of standard geology. [Norton, 2008, p. 28]

But the tempting view (from the standpoint of some confirmation theories) in question is not that the irrelevant conjunct (creation in $4004 \mathrm{BC}$ ) is as well confirmed as the view that the world is old, or as well confirmed as the view that the world is old and looks old (logically equivalent to an irrelevant conjunction, as noted above). The tempting view is that the irrelevant conjunction is as well confirmed as the relevant conjunct, and hence as well confirmed as irrelevant conjunctions with other irrelevant conjuncts. Eagerness to keep the irrelevant conjunct from being confirmed seems to have encouraged momentary confusion of the irrelevant conjunct and the irrelevant conjunction. Perhaps less manifest confusion operates elsewhere. For example, to the extent that I can make sense of Glymour's worry, fear of science's being secretly tainted by Mormonism might be in view. One generally doesn't speak of "reassur[ance]" in the absence of prior doubt. But there is no doubt here: that the irrelevant conjunct is not confirmed is a very simple mathematical theorem.

Especially if one has tacitly retained from the older deductive confirmation theories Hempel's special consequence condition [Hempel, 1965, p. 31], one might be tempted to think that the confirmation of $T_{1} \& T_{2}$ implies confirmation of $T_{2} . T_{2}$ is clearly a deductive consequence of $T_{1} \& T_{2}$, and the special consequence condition implies that an observation that confirms a given sentence also confirms any deductive consequence of that sentence. The special consequence condition is plausible for confirmation viewed as firmness (having a high probability), but not for the now-standard sense of confirmation as incremental increase in firmness (raising the probability). The special consequence condition is neither an axiom nor a theorem of Bayesian confirmation theory, which primarily addresses incremental confirmation. (One can try to capture the idea of firmness in terms of acceptance rules [Swain, 1970], for example, if it seems advisable.) The special consequence condition in fact conflicts with the relevance criterion of confirmation, to the effect that evidence confirms a theory (given background knowledge) iff the theory makes the evidence more probable than it would otherwise be [Mackie, 1969], as well as with Carnap's measure of confirmation [Carnap, 1962, p. 474]. So there is room to hope that Bayesian confirmation of $T_{1} \& T_{2}$ by confirming instances of $T_{1}$ has no confirmatory effect on an irrelevant claim $T_{2}$. Indeed that follows from the mathematics, as noted above.

To sum up, the real problem in the neighborhood, the threat of confirming the irrelevant conjunct, does not happen for the ratio measure (or its rivals, for that matter). The supposedly problematic fact, that the conjunction is

\footnotetext{
${ }^{6}$ The assumption here seems to be that creation in $4004 \mathrm{BC}$ is an irrelevant conjunct. In fact it isn't irrelevant; the probability of creation in $4004 \mathrm{BC}$, given the geological evidence, is famously lower than the prior probability of creation in 4004 BC. $P\left(T_{2} \mid E\right) \neq P\left(T_{2}\right)$. I believe that my treatment above finds the appropriate irrelevant conjunct in taking $T_{2}$ to be inductive historical realism, or perhaps its denial or the like.
} 
confirmed, is true but not problematic; at least no good reason to think it problematic has been provided, while such reasons as have been presented generate an incredible (though toothless) historical skepticism. Typical Bayesian resolutions, which hold that the conjunction is confirmed, but less than the relevant conjunct, are implausible, apparently even to their own proponents (in view of Fitelson's remark about softening the impact [Fitelson, 2002]), and imply (toothless) historical subjectivism about confirmation. By contrast the ratio measure implies that the irrelevant conjunction is confirmed to the same degree as the relevant conjunct, with the irrelevant conjunction getting no confirmation; viewed from the appropriate angle, such results are very natural indeed. Thus the ratio measure has, regarding irrelevant conjunction, advantages but not disadvantages relative to most other measures.

\section{Problem of Measure Sensitivity?}

Such a resolution of the problem of irrelevant conjunction is so perspicuous and reasonable as to suggest that distinctive or unusual features of the ratio measure of confirmation ought to be reconsidered. Fitelson's theorem 3 [Fitelson, 2001], that the conjunction is confirmed less than the relevant conjunct for all considered measures except the ratio measure, I suggest, is a virtue of the ratio measure and hence a clue for addressing his problem of measure sensitivity. I propose applying modus ponens rather than Fitelson's modus tollens to the consequences of choosing the ratio measure. Plausibly, when one finds oneself entertaining an inconsistent belief set, one wants to give up the belief with the least epistemic importance [Gärdenfors, 1984]. In view of the attractions of the ratio measure and the historical skeptical problem for most of the competition, it is appropriate to accept the ratio measure's consequences.

Other distinctive features of $r$ might perhaps be regarded as surprising insights into confirmation, or perhaps a reminder that it is sometimes difficult to make an unambiguous translation of an intuition into a formula [Kuipers, 2000] — which is precisely what is required in order to evaluate a measure of confirmation by its performance in relation to certain supposed desiderata. Recalling that $P(\neg T)=1-P(T)$ as a result of the probability calculus, one should use this identity, and not some independent and somewhat arbitrary intuition, to infer connections between confirmation involving $T$ and confirmation involving $\neg T$, for example. In that case the ratio measure's failure to satisfy a certain negation symmetry between $T$ and $\neg T$ [Fitelson, 2001, Theorem 7, pp. 40, 41] and a related symmetry proposed by Ellery Eells and Fitelson [Eells and Fitelson, 2002] is not worrisome; one ought to derive the behavior under negation from a definition of confirmation and the probability calculus, rather than independently guessing it. Having adopted a highly mathematical notion of degrees of belief and their updating in terms of Bayes' theorem, it is prudent to take the mathematics more seriously than is often done as a guide in defining the degree of confirmation, whether or not one is satisfied with existing 
mathematical arguments such as Milne's. ${ }^{7}$ Perhaps Bayes' theorem also suggests its own notion of confirmation, namely, one directly employed in its "refute and rescale" [Norton, 2007] conditionalization dynamics, the ratio measure (or something equivalent thereto) [Howson, 1983]? Thus Fitelson's problem of measure sensitivity might be an artifact of not recognizing the preferred behavior of the ratio measure.

\section{Bayes' Theorem as a Fractional Linear Trans- formation in $P(T)$ for Hypotheses}

It would be too facile, however, to claim that a surprisingly appropriate treatment of the problem of irrelevant conjunction, combined with deeply problematic results given other measures, provided a decisive reason to take the ratio measure of confirmation to be the one true measure of confirmation in all contexts. That would take either a compelling argument from highly plausible principles, such as Milne aimed to provide, or a weighing of all relevant considerations. Perhaps the idea of a "degree of confirmation" is less meaningful than has often been assumed.

A closer look at Bayes' theorem for hypotheses suggests that the functional dependence of the posterior probability on the prior is not always the same. In contexts where $P(E)$ is not a primitive entity derived from statistical data, but is inferred using the (objective) likelihood $P(E \mid T)$ and the likelihood $P(E \mid \neg T)$ (on which more in a moment), there is also the (subjective) taint of $P(T)$, using the theorem of total probability, in the denominator of $\frac{P(E \mid T)}{P(E)}: P(E)=P(E \mid T) P(T)+P(E \mid \neg T) P(\neg T)[$ Good, 1968, Edwards, 1972, Howson and Urbach, 1993, Royall, 1997, Sober, 2002]. Thus the charming objective linear rescaling of the subjective $P(T)$ as the conditionalization dynamics is illusory in such cases. Instead the result is a fractional linear transformation with $P(T)$ as the variable:

$$
\begin{array}{r}
P(T \mid E)=\frac{P(E \mid T)}{P(E \mid T) P(T)+P(E \mid \neg T)(1-P(T))} P(T)= \\
\frac{P(E \mid T) P(T)}{[P(E \mid T)-P(E \mid \neg T)] P(T)+P(E \mid \neg T)} .
\end{array}
$$

\footnotetext{
${ }^{7}$ An analogy to recent progress in understanding the conservation and localization of gravitational energy in General Relativity comes to mind: by rejecting more or less ad hoc criteria of adequacy and trusting the mathematics to suggest its own interpretation, a protracted and otherwise perhaps insoluble controversy is resolved [Pitts, 2010]. In that context a controversy dating to Schrödinger vs. Einstein in 1918 is resolved by taking seriously the relevant calculations performed no later than 1958 [Bergmann, 1958], once inappropriate separately postulated criteria are rejected. The main casualty is the universal tacit assumption of there being One True Energy, of which every particular expression should be interrelated by a transformation law. Instead there are infinitely many energies, as one might expect from Noether's first theorem in the presence of infinitely many inequivalent time translation symmetries of the laws. One would not expect inequivalent symmetries to yield equivalent conserved quantities.
} 
The choice of primitive quantities is guided by the assumption that one wishes to focus attention as much as possible on $T$, referring to the competition only insofar as the Bayesian formalism requires. A fractional linear transformation is a function of the form $f(x)=\frac{a x+b}{c x+d}$, with $a d-b c \neq 0$ [Churchill and Brown, 1990]. Although such transformations are often encountered in complex analysis, in the present context the variable $x$, being $P(T)$, must be real and take values in the interval $[0,1]$ (or perhaps the open interval $(0,1)$ if $T$ is contingent and a non-dogmatism condition is being enforced). Bayes' theorem enforces $b=0$ : dogmatic rejection of $T$ is never overcome by Bayesian updating. One can make the remaining identifications:

$$
\begin{array}{r}
a=P(E \mid T), \\
c=P(E \mid T)-P(E \mid \neg T), \\
d=P(E \mid \neg T) .
\end{array}
$$

It is clear from the right side of these identifications that only two probabilities determine the three coefficients $a, c$ and $d$. In particular, $c=a-d$, so one has $f(x)=\frac{a x}{(a-d) x+d}$. But two parameters are still one too many, if one doesn't mind extending the positive real numbers by attaching $+\infty$ (much as is done in complex analysis), because one can divide the numerator and the denominator by either $a$ or $d$ and leave only one parameter. The case $d \equiv P(E \mid \neg T)=0$ is roughly falsification of $\neg T$ and hence verification of $T$. In order to have a parameter that takes the value of $+\infty$ for verification of $T$ (to be as similar to the ratio measure as possible), one can divide the numerator and denominator by $d$ :

$$
f(x)=\frac{\frac{a}{d} x}{\left(\frac{a}{d}-1\right) x+1},
$$

or, in terms of more probabilistically meaningful variables,

$$
P(T \mid E)=\frac{\frac{P(E \mid T)}{P(E \mid \neg T)} P(T)}{\left[\frac{P(E \mid T)}{P(E \mid \neg T)}-1\right] P(T)+1} .
$$

The single parameter $\frac{a}{d}=\frac{P(E \mid T)}{P(E \mid \neg T)}$ lies in the range $[0,+\infty]$. The figure illustrates the behavior of this function.

As with the ratio measure, there is a parameter related to confirmation that involves the ratio of two probabilities, and its values run from 0 for (near) falsification of $T$ to $+\infty$ for (near) proof of $T$. But the ratio in question is $\frac{P(E \mid T)}{P(E \mid \neg T)}$ (as also in [Good, 1984]), not $\frac{P(E \mid T)}{P(E)}$, and it produces $P(T \mid E)$ by acting on $P(T)$ in a fashion more complicated than multiplication. Above I argued that a "measure of confirmation" that doesn't keep track of what evidence does to $P(T)$ by Bayes' theorem is a measure that plays little or no useful role. The ratio measure does not have that problem, unlike the competition discussed in debates about the ordinal equivalence or inequivalence of various 


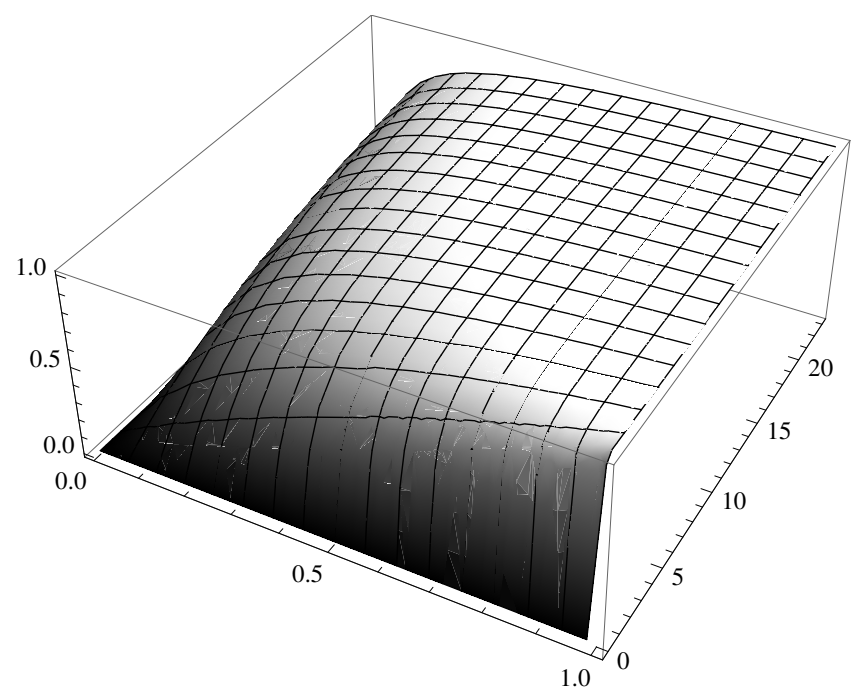

Figure 1: $P(T \mid E)$ as function of $0 \leq P(T) \leq 1$ and $0 \leq P(E \mid T) / P(E \mid \neg T) \leq \infty$, truncated at 23; done with Mathematica.

measures of confirmation [Fitelson, 1999, Atkinson, 2009]. But if confirmation is a transformation that evidence performs on $P(T)$ to give $P(T \mid E)$ by Bayes' theorem, as I propose, then one must be prepared to disentangle the roles of evidence and the prior probability in $P(E)$ in some contexts.

If priors such as $P(T)$ are subjective, then one encounters the further fact that, perhaps disappointingly, $P(E \mid \neg T)$ is itself partly subjective and depends on the priors for a set of mutually exclusive alternatives whose disjunction is $\neg T$ [Good, 1968, Royall, 1997], tainting coefficients $c=a-d$ and $d$. Letting hypotheses $T_{1}, \ldots T_{n}$ form, with $T$, a set of mutually exclusive and exhaustive alternatives, one has

$$
P(E \mid \neg T)=\sum_{i=1}^{n} P\left(E \mid T_{i}\right) \frac{P\left(T_{i}\right)}{1-P(T)} .
$$

One can take $P(T)$ and $n-1$ of the fractions $\frac{P\left(T_{i}\right)}{1-P(T)}$ as independent, in view of the requirement that $P(T)+\sum_{i=1}^{n} P\left(T_{i}\right)=1 . P(E \mid \neg T)$ thus is an average of $n$ objective likelihoods $P\left(E \mid T_{i}\right)$ weighted by $n-1$ subjective ratios of degrees of belief. If one wishes to focus attention on $T$ as far as possible, then bundling all the extraneous likelihoods and priors into $P(E \mid \neg T)$ can be convenient. Likelihoodists [Royall, 1997] might think that a "degree of confirmation" for $T$ by $E$ dependent on (among other things) $n-1$ independent subjective degrees of belief in theories besides $T$, is not worth the trouble, or not worthy to be called scientific. I do not wish here to dispute about the word "scientific." But as human beings, if perhaps not as scientists, often we do have degrees of belief in theories, and the appeal of constructing a world view, even a partly subjective one, makes it seem worthwhile to retain degrees of belief and a measure of confirmation, while frankly admitting the subjectivity of some of its ingredients.

One can consider the problem of irrelevant conjunction while viewing confirmation as a fractional linear transformation (broadly construed so as to 
admit degenerate cases). The quick and easy way to the answer is this: $P\left(T_{1} \& T_{2} \mid E\right)=P\left(T_{1} \mid E\right) P\left(T_{2} \mid T_{1} \& E\right)=P\left(T_{1} \mid E\right) P\left(T_{2}\right)$ using the independence condition $P\left(T_{2} \mid T_{1} \& E\right)=P\left(T_{2}\right)$. One can then expand out the fractional linear transformation involving $P\left(T_{1}\right)$ as above, while for $P\left(T_{2}\right)$ the transformation is just the identity, as one might hope. Thus for the $T_{2}$ transformation, $c=0$ and $a=d$. The fractional linear transformation for $P\left(T_{1} \& T_{2}\right)$ factors into one for $P\left(T_{1}\right)$ and the identity transformation $1 \cdot P\left(T_{2}\right)$, much like the ratio measure factored above.

\section{Acknowledgments}

I thank Robert Audi and some anonymous referees for helpful comments.

\section{References}

[Alexander, 1956] Alexander, H. G., editor (1956). The Leibniz-Clarke Correspondence. Manchester University, Manchester.

[Atkinson, 2009] Atkinson, D. (2009). Confirmation and justification. A commentary on Shogenji's measure. Synthese, pages DOI 10.1007/s11229-0099696-4.

[Ayer, 1952] Ayer, A. J. (1952). Language, Truth and Logic. Dover, New York, second edition.

[Bergmann, 1958] Bergmann, P. G. (1958). Conservation laws in general relativity as the generators of coordinate transformations. Physical Review, 112:287.

[Blumenfeld, 1995] Blumenfeld, D. (1995). Perfection and happiness in the best possible world. In Jolley, N., editor, The Cambridge Companion to Leibniz, pages 382-410. Cambridge University Press.

[Brush, 1982] Brush, S. G. (1982). Finding the age of the Earth: By physics or by faith? Journal of Geological Education, 30:34.

[Burks, 1953] Burks, A. W. (1953). The presupposition theory of induction. Philosophy of Science, 20:177.

[Carnap, 1962] Carnap, R. (1962). Logical Foundations of Probability. University of Chicago, Chicago, second edition.

[Child, 1920] Child, J. M. (1920). The Early Mathematical Manuscripts of Leibniz. Open Court, Chicago.

[Churchill and Brown, 1990] Churchill, R. V. and Brown, J. W. (1990). Complex Variables and Applications. McGraw-Hill, New York, fifth edition. 
[Crupi and Tentori, 2010] Crupi, V. and Tentori, K. (2010). Irrelevant conjunction: Statement and solution of a new paradox. Philosophy of Science, 77:1.

[Cupillari, 2004] Cupillari, A. (2004). Another look at the rules of differentiation. PRIMUS: Problems, Resources, and Issues in Mathematics Undergraduate Studies, 14:193.

[Descartes, 1985] Descartes, R. (1985). Principles of Philosophy, part 3, number 45. In John Cottingham, R. S. and Murdoch, D., editors, The Philosophical Writings of Descartes, Volume I. Cambridge University Press, Cambridge.

[Dorling, 1992] Dorling, J. (1992). Bayesian conditionalization resolves positivist/realist disputes. Journal of Philosophy, 89:362.

[Earman, 1992] Earman, J. (1992). Bayes or Bust? A Critical Examination of Bayesian Confirmation Theory. Massachusetts Institute of Technology Press, Cambridge.

[Edwards, 1972] Edwards, A. W. F. (1972). Likelihood: An Account of the Statistical Concept of Likelihood and Its Application to Scientific Inference. Cambridge University Press, Cambridge.

[Eells and Fitelson, 2002] Eells, E. and Fitelson, B. (2002). Symmetries and asymmetries in evidential support. Philosophical Studies, 107:129.

[Ellenberger and Carozzi, 1999] Ellenberger, F. and Carozzi, M. (1999). History of Geology, Volume 2: The Great Awakening and Its First Fruits -1660-1810. A. A. Balkema, Rotterdam.

[Festa, 1999] Festa, R. (1999). Bayesian confirmation. In Galavotti, M. C. and Pagnini, A., editors, Experience, Reality, and Scientific Explanation: Essays in Honor of Merrilee and Wesley Salmon, pages 55-87. Kluwer Academic, Dordrecht.

[Finch, 1960] Finch, H. A. (1960). Confirming power of observations metricized for decisions among hypotheses, Part II. Philosophy of Science, 27:391.

[Fitelson, 1999] Fitelson, B. (1999). The plurality of Bayesian measures of confirmation and the problem of measure sensitivity. Philosophy of Science, 66:S362.

[Fitelson, 2001] Fitelson, B. (2001). Studies in Bayesian Confirmation Theory. $\mathrm{PhD}$ thesis, Department of Philosophy, University of Wisconsin-Madison.

[Fitelson, 2002] Fitelson, B. (2002). Putting the irrelevance back into the problem of irrelevant conjunction. Philosophy of Science, 69:611.

[Fitelson, 2011] Fitelson, B. (2011). Contrastive Bayesianism. In Blaauw, M., editor, Contrastivism in Philosophy. Routledge. http://fitelson.org/cb.pdf, 07/14/10 draft. 
[Gärdenfors, 1984] Gärdenfors, P. (1984). Epistemic importance and minimal changes of belief. Australasian Journal of Philosophy, 62:136.

[Gillies, 1986] Gillies, D. (1986). In defense of the Popper-Miller argument. Philosophy of Science, 53:110.

[Glymour, 1980] Glymour, C. (1980). Theory and Evidence. Princeton University Press, Princeton.

[Good, 1968] Good, I. J. (1968). Corroboration, explanation, evolving probability, simplicity and a sharpened razor. The British Journal for the Philosophy of Science, 19:123.

[Good, 1984] Good, I. J. (1984). A Bayesian approach in the philosophy of inference: Review of Probability and Evidence by Paul Horwich. The British Journal for the Philosophy of Science, 35:161.

[Gould, 1985] Gould, S. J. (1985). Adam's navel. In The Flamingo's Smile: Reflections in Natural History. Norton, New York.

[Hawthorne and Fitelson, 2004] Hawthorne, J. and Fitelson, B. (2004). Resolving irrelevant conjunction with probabilistic independence. Philosophy of Science, 71:505.

[Hempel, 1959] Hempel, C. G. (1959). The empiricist criterion of meaning. In Ayer, A. J., editor, Logical Positivism, page 108. Free Press, Glencoe, Illinois.

[Hempel, 1965] Hempel, C. G. (1965). Studies in the logic of confirmation. In Aspects of Scientific Explanation and Other Essays in the Philosophy of Science, pages 3-51. The Free Press, New York.

[Hoefer and Rosenberg, 1994] Hoefer, C. and Rosenberg, A. (1994). Empirical equivalence, underdetermination, and systems of the world. Philosophy of Science, 61:592.

[Horwich, 1982] Horwich, P. (1982). Probability and Evidence. Cambridge University Press, Cambridge.

[Horwich, 1983] Horwich, P. (1983). Explanations of irrelevance: On the relative merits of Bayesianism, the hypothetico-deductive method, and Glymour's "bootstrap" theory of confirmation. In Earman, J., editor, Testing Scientific Theories, pages 55-65. University of Minnesota Press, Minneapolis.

[Horwich, 1993] Horwich, P. (1993). Wittgensteinian Bayesianism. In French, P. A., Uehling, Jr., T. E., and Wettstein, H. K., editors, Midwest Studies in Philosophy of Science Volume XVIII, pages 62-77. University of Notre Dame Press, Notre Dame, Indiana.

[Howson, 1983] Howson, C. (1983). Statistical explanation and statistical support. Erkenntnis, 20:61. 
[Howson, 2000] Howson, C. (2000). Hume's Problem: Induction and the Justification of Belief. Clarendon Press, Oxford.

[Howson and Urbach, 1993] Howson, C. and Urbach, P. (1993). Scientific Reasoning: The Bayesian Approach. Open Court, Chicago, second edition.

[Jeffrey, 1980] Jeffrey, R. C., editor (1980). Studies in Inductive Logic and Probability, Volume II. University of California Press, Berkeley.

[Kemeny, 1959] Kemeny, J. G. (1959). A Philosopher Looks at Science. D. Van Nostrand Company, Princeton.

[Kuipers, 2000] Kuipers, T. A. F. (2000). From Instrumentalism to Constructive Realism: On Some Relations between Confirmation, Empirical Progress, and Truth Approximation. Kluwer Academic, Dordrecht.

[Kukla, 1998] Kukla, A. (1998). Studies in Scientific Realism. Oxford University Press, New York.

[Kukla, 2001] Kukla, A. (2001). Theoreticity, underdetermination, and the disregard for bizarre scientific hypotheses. Philosophy of Science, 68:21.

[Laudan and Leplin, 1991] Laudan, L. and Leplin, J. (1991). Empirical equivalence and underdetermination. Journal of Philosophy, 88:449.

[Mackie, 1969] Mackie, J. L. (1969). The relevance criterion of confirmation. The British Journal for the Philosophy of Science, 20:27.

[Maher, 2004] Maher, P. (2004). Bayesianism and irrelevant conjunction. Philosophy of Science, 71:515.

[Milne, 1995] Milne, P. (1995). A Bayesian defence of Popperian science? Analysis, 55:213.

[Milne, 1996] Milne, P. (1996). $\log [\mathrm{P}(\mathrm{h} / \mathrm{eb}) / \mathrm{P}(\mathrm{h} / \mathrm{b})]$ is the one true measure of confirmation. Philosophy of Science, 63:21.

[Norton, 2007] Norton, J. D. (2007). Probability disassembled. The British Journal for the Philosophy of Science, 58:141.

[Norton, 2008] Norton, J. D. (2008). Must evidence underdetermine theory? In Carrier, M., Howard, D., and Kourany, J., editors, The Challenge of the Social and the Pressure of Practice: Science and Values Revisited, pages 1744. University of Pittsburgh Press, Pittsburgh.

[Peirce, 1934] Peirce, C. S. (1934). Eighth Lowell lecture of 1903, "How to theorize". In Hartshorne, C. and Weiss, P., editors, The Collected Papers of Charles Sanders Peirce, volume 5: Pragmatism and Pragmaticism. Harvard University Press, Cambridge. 
[Pitts, 2010] Pitts, J. B. (2010). Gauge-invariant localization of infinitely many gravitational energies from all possible auxiliary structures. General Relativity and Gravitation, 42:601. 0902.1288 [gr-qc].

[Rappaport, 1997] Rappaport, R. (1997). When Geologists Were Historians, 1665-1750. Cornell University Press, Ithaca.

[Reichenbach, 1938] Reichenbach, H. (1938). Experience and Prediction: An Analysis of the Foundations and the Structure of Knowledge. University of Chicago Press, Chicago.

[Rosenkrantz, 1983] Rosenkrantz, R. (1983). Why Glymour is a Bayesian. In Earman, J., editor, Testing Scientific Theories, pages 69-97. University of Minnesota Press, Minneapolis.

[Rosenkrantz, 1994] Rosenkrantz, R. D. (1994). Bayesian confirmation: Paradise regained. The British Journal for the Philosophy of Science, 45:467.

[Royall, 1997] Royall, R. M. (1997). Statistical Evidence: A Likelihood Paradigm. Chapman and Hall, London.

[Russell, 1948] Russell, B. (1948). Human Knowledge: Its Scope and Limits. George Allen and Unwin, London.

[Salmon, 1974] Salmon, W. C. (1974). The pragmatic justification of induction. In Swinburne, R., editor, The Justification of Induction, pages 85-97. Oxford University Press, London.

[Schlesinger, 1995] Schlesinger, G. N. (1995). Measuring degrees of confirmation. Analysis, 55:208.

[Schupbach, 2011] Schupbach, J. (2011). New hope for Shogenji's coherence measure. The British Journal for the Philosophy of Science, 62:125-142.

[Schurz, 2008] Schurz, G. (2008). The meta-inductivist's winning strategy in the prediction game: A new approach to Hume's problem. Philosophy of Science, $75: 278$.

[Shogenji, 1999] Shogenji, T. (1999). Is coherence truth conducive? Analysis, $59: 338-345$.

[Sloan, 1985] Sloan, P. R. (1985). From logical universals to historical individuals: Buffon's idea of biological species. In Atran, S., Burkhardt, Jr., R. W., Corsi, P., Diara, A., Fantini, B., Fischer, J.-L., Hodge, M. J. S., Laurent, G., La Vergata, A., Leikola, A., Louis, P., Mayr, E., Müller, G. H., Rey, R., Roger, J., and Sloan, P. R., editors, Histoire du Concept d'Espèce dans les Sciences de la Vie, pages 101-140. Fondation Singer-Polignac, Paris. 
[Sober, 2002] Sober, E. (2002). Bayesianism-Its scope and limits. In Swinburne, R., editor, Bayes's Theorem: Proceedings of the British Academy, volume 113, pages 21-38. The British Academy and Oxford University Press, Oxford.

[Steel, 2007] Steel, D. (2007). Bayesian confirmation theory and the likelihood principle. Synthese: An International Journal for Epistemology, Methodology and Philosophy of Science, 156:53.

[Swain, 1970] Swain, M., editor (1970). Induction, Acceptance, and Rational Belief. Reidel, Dordrecht. 